Relations industrielles

Industrial Relations

\title{
La politisation des relations du travail
}

Volume 28, numéro 3, 1973

URI : https://id.erudit.org/iderudit/028426ar

DOI : https://doi.org/10.7202/028426ar

Aller au sommaire du numéro

\section{Éditeur(s)}

Département des relations industrielles de l'Université Laval

\section{ISSN}

0034-379X (imprimé)

1703-8138 (numérique)

Découvrir la revue

Citer cet article

(1973). La politisation des relations du travail. Relations industrielles / Industrial Relations, 28(3), 643-663. https://doi.org/10.7202/028426ar
Résumé de l'article

Nous publions ici un résumé des travaux présentés lors du dernier Congrès des relations industrielles de Laval, les 30 avril et 1er mai 1973. Les textes complets paraîtront prochainement dans un ouvrage intituléLa politisation des relations du travail.
Tous droits réservés @ Département des relations industrielles de l'Université Laval, 1973
Ce document est protégé par la loi sur le droit d'auteur. L'utilisation des services d’Érudit (y compris la reproduction) est assujettie à sa politique d'utilisation que vous pouvez consulter en ligne.

https://apropos.erudit.org/fr/usagers/politique-dutilisation/ 


\section{INFORMATION}

\section{La politisation des relations du travail}

Nous publions ici un résumé des travaux présentés lors du dernier Congrès des relations industrielles de Laval, les 30 avril et ler mai 1973. Les textes complets paraîtront prochainement dans un ouvrage intitulé La politisation des relations du travail.

\section{LES FORMES HISTORIQUES DE POLITISATION AU QUÉBEC}

\section{LÉO ROBACK}

Depuis déjà plus d'un an, on parle souvent de la «politisation 》 du syndicalisme et de son action. Au sein des milieux syndicaux comme à l'extérieur, le débat se poursuit. Pour certains, les «chefs》 des organisations syndicales trahiraient leur mandat, et gaspilleraient les fonds syndicaux et les énergies des membres dans une aventure sans issue et sans rapport avec la véritable fonction du syndicalisme. Pour d'autres, le syndicalisme québécois ne fait que commencer à trouver sa vocation réelle en se lançant dans l'action politique visant à «casser le régime », ou, au moins, à amener des réformes radicales au système économique, social et politique dans lequel nous vivons.

Cet affrontement des positions peut paraître caricatural dans la façon dont les tendances opposées sont présentées. Il n'en reste pas moins, cependant, que le débat se situe très souvent en ces termes. Ceci veut dire, donc, que les deux positions s'entendent, implicitement, sur un certain nombre de postulats :

1. L'action syndicale est antinomique : OU revendication dans un système de relations industrielles; $\mathrm{OU}$ visée globale et sociétale.

2. Dans cette antinomie, l'action axée sur les relations du travail, et notamment la négociation collective et l'application de la convention collective exclut, en théorie et dans la pratique, l'action et les orientations axées sur des visées 《sociétales»; à l'opposée, l'action syndicale «politisée» privilégie les objectifs «sociétaux» à long terme par rapport à ceux de la revendication à court terme, et, éventuellement, réduit l'action revendicative au niveau de moyens.

3. Les phénomènes récents du monde du travail au Québec, ceux que l'on qualifie de «politisation», sont nouveaux et inédits dans l'histoire du syndicalisme et des relations du travail chez nous. 
D'abord, même si l'action syndicale paraît antinomique sur le plan TACTCIQUE, celui des moyens, sur le plan STRATÉGIQUE, celui des finalités, l'action est double, multidimensionnelle. Le privilège relatif accordé à l'une ou l'autre dimension, et les formes précises que celles-ci emprunteront dépendront d'un complexe de facteurs, dont la conjoncture économique et politique, dans toute société en particulier à un moment donné.

La première dimension, celle de la revendication d'avantages et de garanties pour les salariés regroupés en syndicat se comprend assez facilement. Mais comment justifie-t-on le postulat de l'autre aspect du syndicalisme, celui qui vise à dépasser la revendication à court terme au nom d'une «utopie» sociétale, d'une image d'une société nouvelle s'inspirant des principes d'une nouvelle répartition du pouvoir et des fruits de l'activité économique, d'un modèle de développement au niveau de la collectivité dans son ensemble?

À cette question, on peut répondre que, historiquement, le syndicalisme, sous quelque forme que ce soit, a toujours véhiculé, ne fut-ce que verbalement, une certaine «utopie» de société différente de celle existante.

$\mathrm{Au}$ Québec, un certain «levain idéologique à caractère «utopique 》 existe dans nos deux plus vieilles tendances syndicales depuis longtemps. Chez les syndicats internationaux et nationaux affiliés à la FTQ, on l'appellerait l'utopie «socialiste », «sociale-démocratie » À la CTCC-CSN, l'utopie sociétale provient de la doctrine sociale de l'Église, à laquelle se greffaient des notions populistes des Chevaliers du Travail (dont quelques syndicats locaux devinrent les premiers éléments du syndicalisme catholique au Québec), et plus tard les idées de Mounier et du «Christianisme de gauche », autant de courants qui débouchaient sur une vision que l'on peut qualifier de «socialisme humaniste», ou d'《humanisme socialiste».

En même temps, l'action syndicale elle-même, dans certaines conditions, secrète aussi le dépassement de son propre projet revendicatif-professionnel, et amène à un jugement sur les structures et le fonctionnement des entreprises, et de là sur le système économique comme tel. Les conditions particulièrement favorables à une telle évolution sont celles où le syndicalisme devient de plus en plus fort, mais où le système économique perd sa vigueur d'antan, c'est-à-dire, est moins capable de concéder les avantages recherchés et revendiqués pour et par les salariés, et/ou n'est plus perçu comme étant capable d'assurer l'avenir de la collectivité populaire dans un modèle de développement acceptable.

Dans cette optique, on pourrait dire que même si le syndicalisme continuait d'accorder un privilège prépondérant, voire exclusif, à l'action revendicative-professionnelle, il y aurait une tendance à regarder de plus en plus vers la société globale et vers l'État, pour la bonne raison que l'évolution d'une société industrielle fait poser de nouveaux problèmes et exigences qui ne sont pas susceptibles de solution par la seule négociation collective au niveau de l'entreprise.

On devrait ajouter aussi la tendance vers une certaine concentration et centralisation de la négociation collective, vers le regroupement des établissements dans une négociation au niveau de l'entreprise (la firme) et celui de plusieurs entreprises 
dans une négociation sectorielle. Ces tendances sont de nature à élargir le domaine des sujets à discuter et à accroître l'enjeu du conflit, d'où une plus grande possibilité d'intervention de la part des autorités publiques au nom de «l'intérêt public ».

\section{LES PRINCIPALES ÉTAPES DES RELATIONS DU TRAVAIL AU QUÉBEC}

Aux fins du présent exposé, nous divisons l'époque moderne en quatre grandes étapes, à savoir :

1. la période avant l'adoption de la Loi des relations ouvrières au début de 1944 ;

2. la période de la «grande noirceur 》, de 1945 à 1959 ;

3. la période de la «Révolution tranquille $\gg$ et de son épilogue, de 1960 à 1970 ;

4. la période du «grand affrontement $\gg$, depuis 1971. Cette période qui n'est pas terminée ne sera touchée ici qu'indirectement.

Dans chacune de ces périodes, nous nous proposons de cerner le type de politisation selon la typologie suivante :

(a) Contenu - finalité

(i) a-politique - l'action politique est tributaire aux objectifs sur le non-partisane) plan de l'action revendicative-professionnelle, et instrumentale à celle-ci :

(ii) politique - l'action politique est axée sur des objectifs inspirés (partisane) d'une idéologie ou d'un projet au niveau de la société dans son ensemble, et qui est normalement associé ou assimilé à une formation politique qui vise à prendre le pouvoir.

(b) Formes - méthode

(i) indirecte

- jeu de pression auprès des autorités, mémoires, lobby, éducation politique pour sensibiliser les syndiqués;

(ii) directe

- prise de position publique sur un gouvernement ou son programme, ou sur un parti politique en particulier.

\section{La période avant 1944}

Les années avant l'établissement du système actuel de règles juridiques sur la reconnaissance et l'exclusivité syndicales et la négociation collective par l'adoption de la Loi des relations ouvrières (3 février 1944) étaient marquées par une situation de marginalité pour le syndicalisme au Québec. Même avant la grande crise des années trente, les syndicats étaient peu nombreux et faibles dans la grande majorité des secteurs économiques. La reconnaissance du syndicat devait être obtenue par la force du syndicat, aucune loi n'obligeant l'employeur à transigner avec une organisation, même majoritaire. Les conventions collectives qui pouvaient exister n'étaient que des «ententes» dont l'application reposait également sur le rapport de forces entre les parties, et encore, ne s'appliquaient qu'aux membres du syndicat contractant. 
La «grande noirceur 》- 1945 à 1959

De nos jours, la période de 1945 à 1959 rappelle surtout quelques événements spectaculaires dans le domaine des relations du travail. L'intervention de la Police provinciale au cours des grèves de Noranda, Valleyfield, Lachute, Asbestos, Louiseville et Murdochville fit la manchette.

Or, il n'y a pas que les grèves et les actions de la police provinciale qui marquèrent cette époque pour le syndicalisme et son action. Il importe de rappeler également l'effet cumulatif d'une série de mesures législatives et, peut-être encore davantage, de l'application des lois ouvrières et sociales par les organismes gouvernementaux avec qui les parties devaient transiger régulièrement, quotidiennement, dans le cadre des activités purement professionnelles. On se réfère ici, bien entendu, à la Commission des relations ouvrières, créée par la Loi de 1944, à la Commission des accidents du travail, au ministère du Travail, et, dans une certaine mesure, au Secrétariat de la Province.

$\mathrm{Au}$ chapitre de la législation, la caractéristique saillante des lois ouvrières de Duplessis est celle de modifier le rapport de force entre l'employeur et la partie syndicale, au détriment de celle-ci. A cet égard, il convient de signaler les restrictions imposées, non seulement au droit de grève, mais aussi au recours à l'arbitrage pour les catégories de salariés déjà privées du droit de grève.

\section{La «Révolution tranquille» et son épilogue (1960-1970)}

Comment caractériser cette période à l'égard de la politisation des relations du travail ? Au risque de simplifier, je propose de souligner les phénomènes suivants :

L'intensification de l'action politique «non-partisane » visant à obtenir de l'État une économie de «règles du jeu » susceptible de favoriser l'action syndicale sur le plan professionnel-revendicatif. Ce mouvement atteindra son comble en 1964 avec les manifestations publiques de la CSN et de la FTQ autour du projet de Code du travail (congrès extraordinaire et menace de grève générale). Le gouvernement Lesage pliera devant les pressions et le nouveau Code consacrera un nouvel équilibre de forces entre les parties patronale et syndicale.

La percée massive et rapide du syndicalisme dans les secteurs public et parapublic où, en même temps, le droit de grève est acquis pour la première fois, fera de l'État un acteur de premier plan dans le domaine des relations du travail, non plus seulement à titre d'《arbitre » ou d'État-législateur et État-administrateur mais en tant qu'État-employeur, tout en conservant les autres attributs. Ceci créera une situation inédite et «politisée » à l'extrême, dans laquelle les armes classiques du syndicalisme (la grève) n'auront plus la même efficacité.

Sur le plan de l'action syndicale «sociétale», par laquelle le syndicalisme cherche à se définir et à se faire définir comme le défenseur des intérêts de la collectivité dans son ensemble, à faire valoir un programme pour réformer les structures économiques et sociales de la société au nom du développement dans le contexte de socialisation, les organisations syndicales se trouveront devancées, en 
quelque sorte, par la «Révolution tranquille», surtout au cours des premières années de cette époque. Ce qui compliquera encore davantage le problème, le nouveau régime et sa nouvelle équipe de jeunes technocrates - au nom de l'«Étatmoteur» - feront valoir précisément les exigences du développement contre les revendications posées par les syndicats à l'État-employeur.

L'action politique syndicale trouvera, dans la «Révolution tranquille», la possibilité d'exaucer son désir de participer, en tant que corps intermédiaire, à des organismes où se prennent des décisions, où des orientations sont arrêtées sur des politiques économiques et sociales touchant toute la collectivité aussi bien que le domaine propre des relations du travail.

Le nœud du problème de la participation, cependant, se situera dans l'équivoque inhérente à la participation, c'est-à-dire, dans le décalage entre les formes de participation et le contenu de celle-ci. Bref, il y aura une vive déception de la part des représentants syndicaux du degré de participation décisionnelle qui leur sera permis .

La «politique de présence» en faveur de la participation, sous quelque forme que ce soit, au processus politique se heurtera de plus en plus aux impératifs de l'action syndicale revendicative, et les conflits dans les secteurs public et parapublic deviendront des «moments privilégiés où l'incompatibilité entre les relations avec l'État-moteur et celles avec l'État-employeur paraîtra de plus en plus difficile à supporter.

Ceci veut dire que cette époque, la période de la «Révolution tranquille», était aussi marquée de conflits «politisés》 dans le secteur privé ; «politisé 》 dans deux sens : (1) dans le sens habituel, soit celui d'une tentative par l'une ou l'autre des parties de faire intervenir l'État pour compenser la faiblesse économique de cette partie. (2) En ce qui regarde le secteur privé, les relations du travail deviennent plus «politisées $\gg$ dans un autre sens, celui que je mentionnai vers le début de cet exposé. On parle maintenant de l'aspect «pouvoir», donc «politique » à l'intérieur de l'entreprise. Il s'agit de l'«empiètement» par le syndicat sur les «droits de gérance $\gg$, de la revendication d'une participation au processus décisionnel au niveau des lieux de travail. Cette revendication varie énormément selon la situation. Elle renferme un éventail de formes et degrés de participation depuis l'information et la consultation jusqu'à la co-décision et au droit de véto sur certaines décisions patronales. Elle porte sur toute une gamme de décisions, insistant sur celles ayant une incidence très directe sur la vie de travail.

$\mathrm{Au}$ cours des dernières années, un certain nombre de conflits dans le secteur privé firent ressortir ce caractère «politique ». Dans le bâtiment, par exemple, et faisant abstraction du facteur de la rivalité inter-syndicale, la sécurité d'emploi fut et demeure, sans doute, l'enjeu central, question qui remet en cause le «droit» de l'employeur d'embaucher et de renvoyer, et qui, en même temps, dans la conjoncture québécoise actuelle, appelle à une réglementation de l'industrie. La sécurité au travail, question à laquelle les travailleurs sont plus sensibilisés qu'auparavant, semble-t-il, tend inévitablement, je le soumets comme hypothèse, vers la revendication syndicale de prise en charge de ce problème par le syndicat, étant donné la défaillance dans ce domaine de l'État. 
On est loin, en regardant ces conflits, des grèves de l'amiante, de Louiseville, de Murdochville, où l'enjeu était celui de la reconnaissance du fait syndical par un patronat paternaliste et autocratique et fort de l'appui d'un gouvernement qui voyait l'action syndicale revendicative comme une espèce de « mutinerie».

\section{L'ÉVOLUTION SOCIO-ÉCONOMIQUE ET LE DÉPLACEMENT DES CENTRES DE POUVOIR}

BERNARD SOLASSE

L'expression «politisation des relations du travail » est foncièrement ambiguë puisqu'elle peut s'entendre comme désignant le caractère ou la dimension politique des rapports qui se nouent entre les différents acteurs impliqués dans le système de relations industrielles ou encore comme l'effet ou la conséquence au niveau du système de relations industrielles d'un phénomène de politisation beaucoup plus vaste et qui lui demeurerait extérieur.

\section{LA POLITISATION DES RELATIONS DU TRAVAIL SES MANIFESTATIONS ET SON ENJEU}

Or, l'élément le plus spectaculaire de la conjoncture actuelle réside incontestablement dans le processus de réorientation idéologique du mouvement syndical québécois et en particulier dans la radicalisation des orientations idéologiques des trois organisations syndicales les plus représentatives soit la CSN, la FTQ et la CEQ.

Au-delà des différences de forme et de fond, la CSN, la FTQ et la CEQ développent une critique de la société actuelle dont les moments forts sont la dénonciation de l'exploitation des travailleurs, la dénonciation du contrôle qu'exercent les forces économiques dominantes sur l'État et sur ses politiques et enfin, la dénonciation du caractère répressif de ce dernier.

Cependant, cette quasi unanimité fait place à une prolifération d'opinions souvent contradictoires dès qu'il s'agit de définir le type de société à bâtir et surtout d'arrêter une conception de l'action syndicale et politique qui devrait permettre d'atteindre ces objectifs. En d'autres termes, le mouvement syndical n'est d'accord ni sur un programme politique ni sur une stratégie politique.

La CSN et la CEQ ont en commun de refuser tout appui officiel à tout parti politique quelqu'il soit. Restent dans les deux cas l'action politique directe, l'information et la formation politique des adhérents, la création de comités d'action politique, la participation aux fronts communs.

La FTQ appuie en principe le NPD avec le succès que l'on sait mais cette stratégie semble difficilement compatible avec les prises de position récentes du Syndicat des métallurgistes. Restent là encore, l'information, l'éducation politique des adhérents, les comités d'action politique et les fronts communs.

Rien par ailleurs n'invite le patronat à ouvrir à grands frais un débat idéologique sur la société actuelle ou sur celle de demain. À ce que je sache, l'éconornie 
du Canada est encore une économie de type capitaliste dominée de surcrô̂t par les firmes multinationales qu'aucune conjonction de forces politiques et syndicales ne menace de l'intérieur pas même le mouvement séparatiste québécois où les éléments socialistes demeurent minoritaires.

Il faudrait conclure en montrant l'incompatibilité des idéologies en présence, l'impossibilité de les concilier. Certains voudraient sans doute qu'on les discutat, qu'on en prit la mesure au nom d'une rationalité dont nous les universitaires serions les dépositaires : le risque serait de leur accorder cette fois une importance qu'elles n'ont pas en tant que discours. De ce rapide survol, je tirerai pour ma part les conclusions suivantes.

Première conclusion: Notre temps n'est plus celui du dialogue. Dialoguer suppose que les interlocuteurs en présence partagent le même langage. Or, il n'y a plus de langage commun, mais des univers clos, séparés, fermés sur eux-mêmes.

Seconde conclusion: Pour avoir longtemps réfléchi sur les idéologies et en particulier sur les idéologies syndicales, j'ai acquis la conviction que l'important n'est pas le contenu, ces visions de soi, de l'autre, de la société qu'elles véhiculent, mais les frustrations qui les génèrent et les aspirations, les attentes, l'espérance qu'elles expriment. Il y a à cet égard, derrière la radicalisation des orientations idéologiques du mouvement syndical québécois, beaucoup plus qu'une simple argumentation d'ordre intellectuel, la force et la capacité mobilisatrice d'une longue accumulation de frustrations.

Qu'il s'agisse de la politisation des conflits ou encore des comités d'action politique syndicaux ou para-syndicaux, je dirai en reprenant les termes employés par Léo Roback que l'enjeu est d'ordre sociétal, que cette politisation n'est pas qu'un simple effet de surface mais qu'elle correspond plutôt à l'émergence de forces nouvelles qui ne se reconnaissent pas dans les partis politiques existant et qui souvent entendent promouvoir une société foncièrement différente de celle que nous connaissons.

L'idée même que le système de relations industrielles puisse avoir une signification politique est propre à faire scandale tant nous sommes habitués à le considérer comme un ensemble de normes et de procédures visant à organiser et à gérer les relations du travail de préférence sur la base de rapports privés entre le syndicat et l'employeur, à l'exception bien sûr du secteur public et para-public.

Or, paradoxalement, c'est dans cette décentralisation des relations du travail voulue par le législateur que réside la fonction politique du système de relations industrielles. En effet, ce morcellement des rapports collectifs du travail a pour effet de freiner le développement des forces centrifuges qui pourrait compromettre le fonctionnement du système économique dans son ensemble et de menacer de l'intérieur la société et son ordre social et politique.

\section{POURQUOI LA POLITISATION DES RELATIONS DU TRAVAIL ?}

Non seulement les groupes sollicitent l'intervention de l'État mais leurs attentes et leurs revendications sont le plus souvent contradictoires. Leur enjeu ce peut être leur sauvegarde en leur survie mais aussi la recherche d'une collaboration de l'État 
qui leur permettra de croître et de prospérer. Ce peut être également lorsqu'il s'agit de nouvelles élites dont le développement valorise les fonctions sociales, la remise en cause des situations acquises, la recherche d'une influence et d'un pouvoir accru.

D'un autre côté, en deça de cette syndicalisation des intérêts se développe un certain nombre de contradictions plus profondes et plus incisives dont l'enjeu est cette fois la remise en cause de l'ordre social lui-même.

C'est à ce type de contradictions que répondent fort maladroitement j'en conviens, ces utopies que nourrit aujourd'hui le mouvement syndical québécois. Elles ne sont souvent que la reprise des exigences qu'avait formulée la classe dominante sans pouvoir les satisfaire : une société plus juste, plus égalitaire, le contrôle du développement économique, la protestation contre l'État de sujétion et de dépendance économique, politique et culturel du Québec. Ces aspirations étaient celles du programme de la «Révolution tranquille»; le mouvement syndical les reprend aujourd'hui à son propre compte, leur confère une forme plus incisive, plus percutante. La radicalisation est à mon avis dans une très large mesure le fruit de l'impatience et de la déception devant les attermoiements des gouvernements qui n'ont pas su ou qui n'ont pas pu réaliser les promesses qu'ils avaient formulées.

\section{L'IMPACT DES SECTEURS PUBLIC ET PARA-PUBLIC SUR LA POLITISATION DES RELATIONS DU TRAVAIL}

JEAN BOIVIN

\section{INTRODUCTION}

Le but de cet exposé est double : d'abord il s'agit d'identifier les éléments qui permettent de conclure à l'existence du phénomène de «politisation» des relations du travail dans le secteur public; ensuite, nous essayerons d'analyser les implications d'un tel phénomène en prenant bien soin d'éviter les jugements normatifs ou moraux sur les bienfaits ou les méfaits de cette «politisation》.

ASPECTS POLITIQUES DE L'ORGANISATION SYNDICALE ET DE

LA NÉGOCIATION COLLECTIVE

On a traditionnellement confiné l'organisation syndicale et la négociation collective telle que pratiquée en Amérique du Nord dans le domaine économico-professionnel. Pourtant, tant l'organisation syndicale que la négociation collective présentent de nombreux aspects qui en font des institutions à caractère politique et ce, même dans le secteur privé de l'économie.

\section{LA POLITISATION DES RELATIONS DU TRAVAIL DANS LE SECTEUR PUBLIC UN PHÉNOMÈNE INÉVITABLE}

La négociation collective dans le secteur public est, par définition, une institution à caractère politique. Nous croyons qu'il en est ainsi principalement à cause de la nature particulière de l'employeur qui est l'État et ce, indépendamment du projet sociétal des organisations syndicales. 
L'un des indices les plus manifestes de la nature politique de la négociation collective dans le secteur public nous est fourni à l'occasion de conflits qui, parce qu'ils portent présumément atteinte à des services jugés essentiels, nécessitent une intervention législative.

Il existe, par ailleurs, au moins deux autres manifestations de la politisation des relations du travail dans le secteur public et qui sont encore une fois liées aux moyens de pression utilisés par les organisations syndicales : il s'agit du «lobbying» et de la participation aux élections pour forcer l'État-employeur à se plier aux exigences des syndiqués. Au Québec, la première méthode a dàjà connu un certain succès tandis que la seconde n'a jamais donné de résultats positifs jusqu'à maintenant pour autant que l'on ait essayé de la mettre à l'épreuve.

Enfin, une autre raison expliquant la politisation des relations du travail dans le secteur public, est la centralisation opérée dans la structure de négociations depuis quelques années.

L'examen des quatre causes ou manifestations de la politisation des relations du travail au Québec qui sont liées, comme nous l'avons vu, à la nature des moyens de pression pouvant être utilisés par les syndicats et à la structure des négociations, nous amène à souligner le cercle vicieux dans lequel se trouvent actuellement les syndicats qui ont à négocier avec l'État provincial : privés de pouvoir de «lobbying 》 efficace et incapables d'exercer des pressions électorales, les syndicats se voient forcés de ne pouvoir recourir qu'à la seule arme disponible aux travailleurs du secteur privé, c'est-à-dire la grève. Cependant, à cause de la centralisation poussée de la structure de négociations, l'exercice pratique du droit de grève est compromis par le fait que le conflit dégénère automatiquement en situation de crise nationale.

La situation des relations du travail dans le secteur public québécois ne serait guère différente de celle prévalant dans les autres Provinces ou États en Amérique du Nord si elle se limitait aux seuls éléments mentionnés plus haut. Mais voilà, comme dans bien d'autres domaines, le Québec sait se distinguer par son originalité. Il possède en effet un mouvement syndical dont les dirigeants ont une idéologie bien particulière qui est celle de la contestation globale du système économique, s'apparentant ainsi au mouvement syndical de certains pays d'Europe.

Dans ce contexte, tout conflit devient l'occasion de souligner la collusion entre l'État et la classe dominante (pour employer un cliché cher à certains sociologues). Toute intervention législative ou judiciaire est l'expression du désir du gouvernement de «tuer le syndicalisme». La logique des tenants d'une telle idéologie est imperturbable : ainsi, même une injonction qui empêche des grévistes de bloquer l'accès des hôpitaux aux ambulances, fait partie de l'arsenal répressif (matraquage est généralement le terme utilisé dans le vocabulaire révolutionnaire).

\section{LES IMPLICATIONS OU LES CONSÉQUENCES DE LA POLITISATION} DES RELATIONS DU TRAVAIL DANS LE SECTEUR PUBLIC

\section{Les conséquences de la négociation collective dans le secteur public sont nécessairement politiques}

Disons d'abord que peu importe la nature des moyens de pression exercés par les syndicats dans le secteur public, les résultats de la négociation collective seront 
nécessairement politiques puisqu'ils influencent la répartition de l'assiette fiscale ainsi que l'orientation des priorités de l'État.

\section{L'utilisation de moyens de pression sur le gouvernement peut conduire d une réaction ou un «back lash》anti-syndical}

Lorsque les syndicats du secteur public tentent de soumettre l'État à des pressions du même genre que celles qu'ils utilisent contre des employeurs du secteur privé comme par exemple, le recours à la grève de durée illimitée, ils peuvent s'attendre à ce que l'État-employeur se transforme rapidement en État-législateur. L'expérience du Québec est assez révélatrice à ce sujet.

La raison de ce volte-face de l'État-employeur est bien simple: la pression exercée par les syndicats au moyen de grèves à durée illimitée ne s'exerce pas tellement sur l'État-employeur comme sur la population qui n'est même pas partie au conflit. Dans la mesure où les services touchés causent de sérieux inconvénients à celle-ci, comme dans le cas des hôpitaux, l'État-législateur sera soumis à d'autres pressions, cette fois-ci exercées par la population qui désire le rétablissement de ces services.

Lorsque de tels conflits ont tendance à se répéter assez fréquemment comme en témoigne les nombreux Bills $(25,1,19,73)$ passés au Québec pour rétablir des services dits essentiels, il n'est pas surprenant de voir l'État tenter d'adopter des législations anti-syndicales à caractère permanent comme c'est le cas avec le projet de loi no 89 soumis à l'Assemblée nationale en décembre 1972.

Les conséquences de la politisation des relations du travail sur le régime de négociations collectives

Il est permis de croire que les qualités qui font de la négociation collective un excellent mécanisme pour solutionner les différends dans le secteur privé ne se retrouvent à peu près pas dans le secteur public. Cette situation existe parce que la nature fondamentalement politique des relations du travail dans le secteur public est incompatible avec le modèle de négociation collective développé dans le secteur privé. (On n'a qu'à penser au fait que plusieurs des gains importants réalisés par les syndicats sont obtenus dans une situation de crise après que le législateur soit intervenu. Si c'est là la seule façon pour les syndicats de faire triompher leur point de vue durant les négociations, il faut conclure qu'il y a quelque chose qui cloche dans le régime actuel de négociation collective).

Il appartient maintenant aux experts en relations industrielles de cesser de vanter l'institution qu'est la négociation collective telle que développée dans le secteur privé comme la seule façon valable pouvant permettre une participation effective des travailleurs à la détermination des conditions de travail et de chercher de nouvelles approches qui tiendront compte de la nature particulière de l'État et du caractère fortement politisé des relations du travail dans le secteur public. 


\section{LA PHILOSOPHIE DU CODE DU TRAVAIL}

\section{JEAN-RÉAL CARDiN}

Il faut rappeler au départ que le droit du travail, en Amérique du Nord comme dans les pays occidentaux industrialisés en général, est issu directement du libéralisme économique. Il en partage, dans sa philosophie de base, les principaux postulats en dépit ou plutôt à cause du fait même qu'il ait été traditionnellement considéré par le législateur comme un droit d'exception au droit commun, lui-même le reflet fidèle de l'idéologie dominante véhiculée historiquement par ce système de valeurs.

Tout en faisant du droit du travail un droit d'exception au droit commun afin de faire place, entre autres choses, aux réalités collectives que ce dernier ignorait systématiquement, le législateur des pays occidentaux, et peut-être plus particulièrement celui d'Amérique du Nord, a en quelque sorte consacré dans ce droit du travail lui-même les pouvoirs et les rôles dévolus aux partenaires sociaux par la société libérale traditionnelle.

Ainsi, tout en proclamant le droit d'association des travailleurs pour fins de négociations collectives et de protection, grâce à l'organisation des intérêts individuels de ces derniers, on a, en même temps, limité les groupes auxquels ce droit était octroyé. On a, par le fait même, non seulement considéré ce droit comme une espèce de privilège concédé à certaines conditions, mais encore s'est-on trouvé à réitérer, dans les lois du travail, le rôle d'initiateur incontesté du propriétaire des moyens de production, de l'entreprise, quant à la gouverne de la chose économique aux plans de la décision et de l'administration.

Tout ce que notre Code du travail, expression fidèle de cette philosophie, proclame donc, c'est la volonté de permettre un meilleur rapport de forces au sein de l'entreprise, en permettant, par l'association des salariés, par la négociation et la convention collectives, par la procédure de règlement des griefs, etc. un meilleur contrôle des décisions patronales, par les salariés, sur certains aspects seulement de ces décisions, sans entamer pour autant la source du pouvoir d'où elles émanent.

Même si, en sociologie, on reconnaît au syndicalisme un double rôle d'agent de revendication professionnelle pour ses membres sur le marché du travail et d'agent de transformation sociale à plus ou moins long terme à l'avantage de l'ensemble des travailleurs salariés dans une communauté politique donnée, nos lois du travail, dans leur philosophie et dans leur économie, ne reconnaissent explicitement, et n'organisent que le premier de ces deux rôles dévolus au syndicalisme. Quant au second de ces rôles, tout au plus permettent-ils à ce dernier de rechercher corollairement une amélioration, je dirais quantitative, de la situation des salariés à l'intérieur des structures établies, mais jamais une transformation en profondeur de ces structures elles-mêmes.

La convention collective, telle qu'élaborée par nos lois du travail surtout à partir des années ' 40 , et «offerte» au monde syndical par les pouvoirs publics au moyen de l'accréditation et de l'agence exclusive de négociation, a fait du syndicat, légalement, une institution a-politique, greffée essentiellement sur l'entreprise ou même l'établissement, et vouée presque par la force des choses à une pure 
action de contrôle et de revendication limitée aux seuls plans des intérêts professionnels de ses membres considérés en tant que salariés de cette entreprise.

Ce n'est pas à dire que le mouvemnt syndical n'a pas débordé ce rôle dans l'expression de sa personnalité à certaines périodes de son histoire ; ce que je veux dire, c'est que le Code du travail, dans ses institutions mêmes, confine le syndicalisme au rôle que je viens de décrire.

Il faut reconnaître qu'historiquement, étant donné l'hégémonie des valeurs du capitalisme libéral en Amérique du Nord, cet «isolement» de nos systèmes de relations industrielles, et donc de notre syndicalisme, de la chose politique a été accepté comme allant pratiquement de soi, aussi bien par la population en général que par les travailleurs salariés et les associations syndicales elles-mêmes.

Ce rôle a-politique du syndicalisme nord-américain, cet «économisme 》 dans lequel nos législations du travail le confinent, cette qualité de «chien de garde» d'un patronat local par ailleurs souverain, est tellement ancrée dans nos mœurs sociales que, même si la société libérale n'est plus ce qu'elle était et que ses facteurs nouveaux, (que nous verrons plus loin) appellent une définition renouvelée des rôles respectifs des agents en relations du travail, on ne peut, dans plusieurs milieux, imaginer d'autres rôles pour les syndicats que ceux que la loi leur a historiquement concédés.

Remarquons que, même si depuis 1965 , le 'Code du travail inclut les pouvoirs publics dans sa définition d'《employeur », toute l'économie du Code a été originellement conçue en regard du secteur privé de l'économie. C'est une des raisons, à mon avis, des difficultés très considérables qu'éprouvent nos démocraties libérales nord-américaines à inventer les structures juridiques des rapports collectifs de travail dans les secteurs publics d'emploi où l'État a consenti à s'abaisser au rang d'employeur un peu «comme les autres».

\section{LES FACTEURS D'ÉVOLUTION ET DE REMISE EN QUESTION DE CETTE « PHILOSOPHIE 》}

\section{Les relations du travail face aux aspirations des «nouveaux syndiqués»}

Les changements dans les structures d'emploi, l'importance accrue des cols blancs en général, des semi-professionnels et des professionnels ainsi que des cadres de toutes dénominations, de même que l'extension de la formule syndicale à ces «nouvelles» catégories de salariés semblent nous révéler que les structures de pouvoir et les mécanismes de relations collectives consacrés dans les dispositions du Code du travail ne répondent pas ou répondent mal aux caractéristiques et aux aspirations de ces groupes de salariés. Un ingénieur, un travailleur social, un professeur d'université, un enseignant en général, un médecin, un journaliste professionnel, etc. ne peuvent, dans l'exercice même de leurs fonctions, pas plus que dans leurs rapports avec les directions qui les emploient, se contenter, à l'instar des cols bleus de l'industrie, de la distinction étanche qui est faite par notre droit $\mathrm{du}$ travail entre les fonctions de direction d'une part, et celles de pure exécution d'autre part. 
Je ne veux pas monter en épingle indûment ces inadaptations ou ces difficultés de nos lois du travail actuelles face à ces problèmes relativement nouveaux que provoque l'avénement au syndicalisme de groupes de salariés et d'institutions qui souffrent mal les vieilles catégories juridiques et les structures de pouvoir consacrées par ces lois. Je ne prétends pas que les solutions sont faciles à trouver, quel que soit d'ailleurs le type de société auquel on a affaire, ni qu'il soit approprié de vouloir trop rapidement ou trop radicalement les délaisser pour en adopter de nouvelles. Je constate simplement que leurs déficiences contribuent à les discréditer aux yeux des intéressés et à inciter ces derniers à chercher la solution à leurs problèmes en dehors de ces législations, à remettre en cause le système de valeurs même qui les supporte. Elles les justifient d'avoir recours à des modes d'actions extra-légaux ou franchement illégaux qui contribuent, par le fait même, à «politiser» les situations en cause.

\section{Les relations du travail a l'heure des choix politiques}

Un deuxième facteur d'évolution de notre société qui tend à remettre en question le caractère fonctionnel de nos lois du travail, c'est ce que j'appellerais la fin de l'autonomie traditionnelle des systèmes de relations industrielles par rapport à l'administration de la chose publique, à la fonction politique dans son sens le plus large et aux décisions communautaires qui sont les siennes désormais. Les systèmes de relations industrielles sont de plus en plus liés organiquement aux choix politiques et aux décisions qui en émanent sous forme de programmes de toutes sortes en matière économique et sociale. C'est là le résultat net de la socialisation des économies, de l'interdépendance accrue des centres de décision autrefois autonomes, et du rôle sans cesse croissant de l'État et de ses agences dans les fonctions vitales des sociétés contemporaines.

Notre Code du travail est issu du libéralisme économique. Il consacre dans ses dispositions des valeurs et des institutions issues d'un laisser-faire collectif qui répondent mal, sinon pas du tout, aux exigences nouvelles que lui impose cette société.

La convention collective telle que définie dans le Code, avec les pouvoirs respectifs qu'elle consacre de la part des parties négociatrices, avec la notion de «condition de travail » qu'elle utilise, avec le cadre étroit de l'unité de négociation qui est le sien, ne peut actuellement répondre d'une façon valable aux exigences d'une politique de main-d'œuvre cohérente. De nouvelles lois (ex : la Loi sur la formation et la qualification professionnelle de la main-d'oeuvre) et de nouveaux mécanismes (comités de reclassement, etc.) doivent venir supplémenter et même remplacer les dispositions du Code $d u$ travail car ce dernier ne peut absorber ou régler seul les tensions créées par ces problèmes. Et même ces outils nouveaux étant encore très imparfaits, la nouvelle conscience sociale transpose alors les données du problème dans leur dimension communautaire et cherche alors à les résoudre politiquement : le système lui-même est mis en cause et les mécanismes classiques de solutions prévues par nos lois du travail: grève professionnelle, piquetage symbolique, ont tendance à être délaissés comme étant inutiles et hypocrites pour faire place aux manifestations de masse, à la mobilisation de l'opinion publique, à l'occupation des lieux de travail, à l'appel aux solidarités extérieures, etc. 
Un dernier exemple, un des plus représentatifs de notre propos, c'est l'incapacité, pour nos lois du travail, de permettre une pénétration syndicale plus étendue chez les plus démunis des travailleurs salariés. Le concept de l'accréditation localisée fondé sur l'adhésion majoritaire absolue des groupes requérants, empêche les organisations syndicales existantes de pénétrer ces secteurs de main-d'œuvre, et frustre les salariés impliqués des droits collectifs et des protections qui, pourtant, sont supposément offertes à tous les «salariés » par notre Code du travail.

Les centrales syndicales, outre qu'elles sont conscientes des limites même de leur action traditionnelle dans les cadres juridiques existants, développent une mauvaise conscience à l'endroit des laissés pour compte du système et ont tendance à se donner de nouveaux objectifs, à entreprendre des actions inédites, à «politiser» donc le débat en débordant de l'action de type professionnel et en «radicalisant» leur action pour s'attaquer au système économique et social lui-même.

\section{Les relations du travail et l'État-employeur}

Un troisième élément d'évolution qui fait éclater les schèmes traditionnels de nos lois du travail, c'est l'avènement des pouvoirs publics comme employeur le plus important de la collectivité. Le fait que l'État en donnant accès à la négociation collective à ses propres employés et en devenant, en dépit des ambiguités juridiques déjà soulignées plus haut, l'employeur ultime et réel de ceux qui dépendent de lui dans la détermination de leurs salaires et de leurs conditions de travail (fonction para-publique), a contribué par la force des choses à «politiser», non seulement pour les groupes impliqués, mais pour l'ensemble du système de relations industrielles des questions qui, selon l'esprit de nos lois du travail, sont d'ordre purement professionnel et catégoriel.

Toutes ces critiques ne se veulent que des constatations. Je ne saurais à partir de là, conclure que ces déficiences sont facilement corrigibles ni que les corrections seraient nécessairement meilleures que ce qui existe présentement. Tout ce que je retiens de ceci, c'est que, pour reprendre les expressions des organisateurs de ce congrès, l'isolement de l'«industriel» du «politique», que nos démocraties nord-américaines ont réussi à établir jusqu'aux années récentes, disparaît graduellement. En conséquence, la politisation «a-sociétale» (qui à mon avis est plutôt l'absence de politisation) tend à faire place, par la force de l'évolution sociale, à la politisation «sociétale \i.e. à la politisation tout court des problèmes de relations industrielles.

Les lois du travail sont-elles alors elles-mêmes un facteur de politisation, de par leur caractère dysfonctionnel en économie moderne, ou ne sont-elles plutôt qu'à la remorque d'une mutation de l'idéologie de la société globale ?

Je crois, pour un, qu'elles sont ces deux choses à la fois. Les déficiences que j'ai tenté d'illustrer contribuent sûrement à la politisation de nos relations du travail. Jusqu'à quel point et dans quelle mesure peuvent-elles être corrigées sans supposer un système économique totalement différent de celui qui est le nôtre à l'heure actuelle ? La réponse à cette question est justement celle qui dépend de cette mutation idéologique communautaire et du caractère, réformateur ou révolutionnaire, qu'elle prendra dans les années qui viennent. 


\section{LES LIMITES DU NÉGOCIABLE ET LE DÉBORDEMENT DES CONFLITS}

André Thibaudeau

Il est assez difficile de lire le titre de ma conférence sans réfléchir aux nombreuses luttes que le mouvement syndical a livrées ces dernières années et à celles que les travailleurs ont dû subir dans le passé. Les objectifs pour lesquels les luttes d'Asbestos, de Louiseville, de Murdochville, etc., ont été menées et ceux qui ont motivé les derniers conflits auxquels nous avons assisté ne sont pas les mêmes. Il y a dix ans, quinze ans ou vingt ans, on se battait pour obtenir des salaires décents, des heures de travail et des vacances acceptables. On se battait aussi pour la reconnaissance des syndicats par le patronat.

\section{RECONNAISSANCE SYNDICALE ET NÉGOCIATION : UNE LENTE ÉVOLUTION}

Notre loi obligeant le patronat à négocier et non à conclure une convention collective, il était assez fréquent de ne pas pouvoir conclure une première convention collective de travail pour un groupe donné, même si on avait réussi à obtenir une accréditation. Ceci avait pour effet de déclencher des grèves que le patronat gagnait par l'usure, tuant par le fait même le syndicat. Si le syndicat n'était pas suffisamment fort pour faire la grève, il disparaissait de toute façon avec le temps. Aujourd'hui, ce genre de lutte est plutôt rare. Généralement, un syndicat qui, aujourd'hui, obtient une accréditation signe une première convention collective et, comme le patronat reconnaît le syndicat, il accorde assez facilement la retenue syndicale et même l'établissement de la «Formule Rand».

La reconnaissance syndicale étant acquise, les parties ont fini par établir une technique de négociation. Par exemple : d'un groupe à l'autre, la négociation d'une convention collective de travail se faisait et se fait encore, dans bien des milieux, par voie de comparaisons.

Les bureaux de recherche syndicaux qu'on a vu naître au cours des dernières décennies ont facilité cette tâche en se spécialisant dans la préparation de tableaux comparatifs de salaires et de conditions de travail. La méthode des comparaisons, à elle seule, offrait donc largement matière à négociation.

De plus, ici et là, d'autres types de clauses commençaient à apparaître à la convention collective. Au cours de ma carrière syndicale, j'ai en effet remarqué que les négociateurs syndicaux, moi comme les autres, cherchaient à faire passer certaines idées nouvelles chez les employeurs plus complaisants ou aux prises avec une situation économique qui les empêchait de faire face à une lutte, ou encore chez des employeurs à l'esprit plus ouvert au point de vue social. Il était donc à prévoir que, de cette façon, des gains nouveaux s'obtiennent et servent ensuite de modèles pour d'autres négociations.

\section{LE CONTRÔLE SYNDICAL : CONTRAINTES ET LIMITES}

Sans aborder directement le problème des changements technologiques, certains syndicats ont commencé à négocier timidement certaines clauses dont plusieurs ne réglaient pas nécessairement le problème, à savoir : des indemnités de fin d'emploi 
et d'autres clauses ne pouvant que partiellement et trop lentement diminuer l'impact de ces changements comme par exemple : diminution des heures de travail, interdiction à l'entreprise de donner des contrats à forfait, etc. On ne peut pas dire que ce genre de clauses visant à enrayer les effets nocifs des changements technologiques, comme les licenciements, ont été tellement répandues si ce n'est dans le secteur public.

Durant bien des années, ceci était compréhensible puisqu'on se battait surtout pour le pain et le beurre, mais un certain niveau de confort atteint, on n'a pas su, de part et d'autre, faire des prévisions, ayant pris l'habitude de ne parler que du présent. Par l'usage, ceci a fixé une limite au négociable, les mêmes thèmes revenant toujours d'une convention collective à l'autre.

Il n'en demeure pas moins que, maintenant, c'est par dizaines de milliers que des travailleurs perdent leur emploi, sont déplacés ou deviennent chômeurs et assistés sociaux. Quelles que soient les politiques d'une compagnie vis-à-vis les changements technologiques, les salariés n'ont guerre de contrôle sur ces phénomènes puisqu'on n'a pas encore attaqué d'une façon réelle les droits de la gérance et qu'aucune forme de cogestion sérieuse n'a été entreprise.

$\mathrm{Du}$ reste, ces moyens seraient-ils de nature à résoudre un tel problème ? Je ne le crois pas, car il n'y a pas que les changements technologiques qui limitent ou diminuent l'emploi et il est quasi impossible d'enrayer les autres causes de chômage à la table de négociation. Selon moi, il s'agit d'une responsabilité qui incombe à l'État, puisqu'il est le seul à y pouvoir vraiment quelque chose.

Animé par le même esprit que dans l'entreprise privée et ayant en tête les mêmes objectifs, c'est-à-dire l'obtention de meilleures conditions de salaire et de travail, le syndicalisme a envahi le secteur public. On peut diviser cette période en deux étapes. Dans un premier temps, le secteur public s'est comporté exactement comme un employeur de l'entreprise privée et a négocié ses conventions collectives point par point, ne concédant qu'étape par étape et se durcissant seulement sur certaines demandes, le point de concession étant le plus souvent déterminé en fonction du niveau acceptable de taxes que l'on pouvait faire avaler au contribuable.

À la deuxième étape, comme chacun sait, une nouvelle limite a été fixxée au négociable dans le secteur public et para-public: la politique salariale du gouvernement. Cette limite est une conséquence directe de la syndicalisation des fonctionnaires. Elle tient aussi au fait que, depuis que le gouvernement, par le Bill 46, peut exercer son droit de regard dans les services publics et para-publics, il pose comme principe que seuls les élus du peuple peuvent décider du budget et que, par voie de conséquence, la politique salariale n'est pas négociable.

À ceci, les travailleurs ne pouvaient pas ne pas réagir car, traditionnellement, cette limite du négociable n'avait jamais été acceptée. Or, les travailleurs n'acceptent pas de gaieté de cœur de perdre ce qu'ils considèrent comme un droit acquis et pour le conserver, ils sont prêts à recourir à la grève.

Le phénomène économique, pris dans son ensemble, devient politique car il est la résultante de choix politiques. Ainsi, le facteur économique qui constituait 
une force de négociation est radicalement changé par des impératifs politiques plutôt qu'économiques. Le mouvement syndical, malgré toutes les difficultés qu'il devait surmonter, a donc décidé de former un front commun dans le secteur public afin d'être en mesure de discuter avec la seule autorité compétente, le gouvernement qui était devenu le véritable centre de décision.

\section{LE SYNDICALISME ET LA DÉFENSE DU STATUT SOCIAL DU TRAVAILlEUR}

Cependant, il est prouvé, et l'exemple du secteur public ne fait que le confirmer de façon éclatante, que certains problèmes fondamentaux touchant le statut des travailleurs comme salariés et encore moins leur statut de citoyens ne peuvent être réglés au niveau de l'établissement et qu'à ce niveau la négociation devient de moins en moins efficace parce qu'elle ne fait qu'accentuer les divergences d'intérêts qui séparent les différents groupes de travailleurs. Comme les employés en général subissent les effets des politiques gouvernementales et les effets de la fonction économique de l'entreprise, surtout des grandes, ils ne pourront pas, dans notre système, établir et protéger leur statut social de travailleurs uniquement à la table de négociation, ce qui veut dire que le mouvement syndical, face à des impératifs qui le frappent, devra modifier sérieusement ses structures (et ceci ne sera pas facile) afin de défendre les intérêts de ses membres là où leur sort se décide, c'est-à-dire, là où les politiques économiques et sociales s'établissent.

De nouveaux mécanismes devront aussi être prévus car, si, au niveau de l'établissement, il est possible de faire des concessions de part et d'autre à la veille d'une grève ou pendant qu'elle a lieu, dans le secteur public cela n'est guère possible une fois que le budget du gouvernement est adopté ou décidé unilatéralement.

CONCLUSION

Pour conclure, il m'apparaît clair que, si la convention collective peut encore protéger et améliorer le statut du salarié, elle ne peut rien pour celui du travailleur. Les syndicats sont impuissants à concilier des intérêts aussi contraires que ceux qui sont rattachés à la fonction économique de l'entreprise et ceux des travailleurs à la recherche d'un statut social acceptable.

\section{LES CONSÉQUENCES DE LA POLITISATION DES RELATIONS DU TRAVAIL}

\section{GÉRARD DION}

La politisation des relations du travail est une chose autre que la politisation du syndicalisme. Il y a cependant interrelation entre les deux. D'une part, certaines formes de politisation des syndicats entraînent la politisation des relations du travail et, d'autre part, certaines formes de politisation des relations du travail entraînent à leur tour la politisation des partenaires dans le système des relations professionnelles dont les syndicats.

Par conséquent, si on est amené à traiter de la politisation des syndicats, c'est seulement en tant qu'elle affecte le régime des relations du travail et le syndicalisme 
lui-même qui est une organisation indispensable dans une société industrielle que l'on veut démocratique.

\section{LA POLITISATION}

Tous les faits sociaux sont potentiellement politiques; ils sont plus ou moins chargés de politique, car l'univers politique relève d'un type de relations et non de faits. Le problème fondamental est alors d'apprécier la densité politique dont se charge une relation sociale jusqu'à devenir une relation politique. Il n'y a pas de frontière fixée ne varietur: le politique n'est que du social qualifié d'une certaine façon. Selon les époques et selon le contexte culturel, une masse de relations peuvent ou non être plus ou moins politisées.

La politisation prend des formes multiples et parfois subtiles. Selon le type de politisation et la nature de l'idéologie qu'elle porte, les conséquences ne seront pas les mêmes.

Lorsque les partenaires réussissent à régler leurs problèmes entre eux, même à la suite d'affrontements, sans faire intervenir le pouvoir politique, il n'y a pas de politisation.

L'action politique que peuvent mener les partenaires dans le champ politique ne politise pas nécessairement les relations du travail, à moins qu'elle ait des répercussions sur leurs rapports mutuels dans le champ même des relations du travail. Ainsi, que le syndicat des travailleurs-unis de l'automobile accorde son appui au Nouveau parti démocratique ne politise pas pour autant les relations qu'il entretient avec les fabricants d'automobiles dans la négociation collective et l'administration de la convention. Et il en vas de même pour l'appui que peuvent fournir les fabricants d'automobiles aux partis libéral, conservateur ou créditiste.

\section{COMMENT SE POLITISENT LES RELATIONS DU TRAVAIL}

L'origine de la politisation des relations du travail tient à deux ordres de causes : la nature des choses et la volonté systématique des personnes.

\section{La nature des choses}

L'évolution du régime économique qui se manifeste par un dépérissement graduel de l'économie de marché au profit d'une planification entraîne, d'une part, une intervention plus grande des pouvoirs politiques dans le champ des relations du travail et, d'autre part, la création au niveau de l'État d'une multitude de comités, de conseils, de commissions aux rôles variés allant de la participation à la détermination des objectifs des politiques économico-sociales à l'élaboration des décisions et même jusqu'à l'administration. Dans ces organismes publics qui exercent des fonctions vraiment politiques, les partenaires sociaux sont représentés en tant que forces organisées et représentatives œuvrant dans le champ des relations du travail.

D'un côté, l'action professionnelle des syndicats qui porte sur la défense des droits et la promotion des intérêts des travailleurs partout où ils sont en cause prend parfois un caractère politique et débouche sur la politique. D'un autre côté, l'action menée par les syndicats vise des objectifs plus généraux comme l'aménagement des 
rapports dans la société globale. Dans le premier cas, cette action lui est propre et lorsqu'elle en vient à toucher la politique, elle est le prolongement naturel de l'action professionnelle. Dans le second, elle est commune à tous les groupes sociaux et devient plus ou moins politisée selon l'idéologie prônée et les stratégies utilisées pour amener une transformation sociale. Il est toutefois important de noter qu'alors ce ne sont pas tant les visées de changements qualitatifs en eux-mêmes qui politisent mais bien les méthodes d'action employées pour y arriver.

\section{Volonté systématique des personnes}

Cette politisation se produit d'une façon générale lorsque, dans le but d'infléchir le fonctionnement normal du régime des relations du travail on fait appel à des normes idéologiques qui lui sont étrangères ou que l'on y entraîne constamment l'intervention des pouvoirs politiques.

Elle se produit d'une façon particulière quand on utilise les relations du travail elles-mêmes pour des fins d'ordre strictement politique. Les relations du travail deviennent tantôt un champ privilégié où l'on mène des batailles politiques, tantôt une occasion ou un moyen pour réaliser des objectifs qui se situent au niveau de la société globale. Ainsi, au lieu de se servir des voies ordinaires et des mécanismes institutionnels d'expression et de pression pour gagner à sa cause le public et influencer la politique on utilise les conflits du travail existant et on ira même jusqu'à en susciter.

Cette politisation voulue et systématique peut-être causée aussi bien par les gouvernants eux-mêmes que par les syndicats, les employeurs ou d'autres agents extérieurs au monde des relations du travail comme les agitateurs. Même les média d'information peuvent aussi politiser les relations du travail en présentant les événements de manière que le public les perçoive sous un angle politique.

\section{LES CONSÉQUENCES DE LA POLITISATION}

Toutes les formes de politisation des relations du travail n'ont pas la même portée ni les mêmes conséquences.

La politisation qui découle de la transformation du régime économique avec l'intervention grandissante du rôle de l'État dans tous les domaines peut conduire, si on n'y fait pas attention, à une dégradation de l'autonomie du système des relations professionnelles de même qu'à une diminution de la latitude de décision attribuée aux organisations de travailleurs, au patronat et aux services officiels spécialisés. Elle peut encore altérer la négociation collective qui est le pivôt de notre système de relations professionnelles.

Par ailleurs, si la complexité des problèmes conduit l'État à intervenir de plus en plus dans l'ensemble de la vie économique et sociale, c'est-à-dire sur le terrain des activités professionnelles et syndicales, il est normal que des liens nouveaux cherchent à s'établir entre les pouvoirs publics et les organisations professionnelles pour la préparation et l'élaboration des politiques économiques et sociales. D'une manière 
ou d'une autre, la rencontre et la concertation entre les instances politiques et les représentants des divers intérêts professionnels s'imposent, car on est condamné à la participation.

Les conséquences de la politique des relations du travail provenant de la volonté des personnes ou des groupes qui utilisent ces relations pour des fins politiques, sont celles qui semblent les plus menaçantes.

Cette politisation est contagieuse. Du moment qu'elle est introduite quelque part, par qui que ce soit, tout le monde est obligé de la subir. Même l'action entreprise pour la neutraliser ou l'extirper devient à son tour politique.

\section{POlitisation DES RELATIONS DU TRAVAIL ET POLITISATION SYNDICALE}

Il existe une certaine cohérence entre le régime des relations du travail et le régime syndical que nous avons et qui ont été sanctionnés par la législation au Canada et au Québec depuis 1944.

Ce régime juridique et contractuel postule un certain apolitisme syndical. En effet, si le syndicat représente obligatoirement tous les travailleurs d'une unité de négociation et si presque partout les travailleurs doivent adhérer au syndicat ou le supporter financièrement, il faut au nom de la liberté d'association et de la liberté d'opinion que le syndicat leur soit accessible en ne portant pas atteinte à leur liberté de conscience ni à leur liberté civique. Comment peut-on alors imposer aux travailleurs l'appartenance et le support financier à un syndicat qui véhiculerait une idéologie politique qui répugnerait à leur conscience ou à leurs convictions ? Au fond, c'est toute l'argumentation qui a été développée, il y a une quinzaine d'années, et qui a amené la déconfessionnalisation de la CTCC.

Les conséquences apparaissent assez graves tant sur la syndicalisation que sur le régime même des relations du travail.

Il faudra, en premier lieu, faire disparaître des conventions collectives les clauses d'adhésion obligatoire au syndicat de même que celles qui imposent aux travailleurs un support financier, comme la formule Rand. Le syndicalisme ne comptant que sur ses propres moyens en ressortira grandement affaibli.

Il faudra ensuite modifier substantiellement notre régime de négociation collective et l'ajuster à une nouvelle forme de pluralisme syndical. Il faudra donc écarter le monopole de représentation dans une unité de négociation et y accepter le pluralisme syndical au niveau des établissements.

Il y a donc un type de politisation que les syndicats doivent s'interdire sous peine de chambarder totalement notre régime syndical et notre régime de négociation collective. Si les syndicats veulent s'y livrer, c'est à eux de décider et à eux seuls. Mais ils doivent en accepter les conséquences, car ils ne pourront pas à la fois bénéficier des avantages du régime actuel et en même temps refuser les limitations qu'il impose. 
Le syndicalisme qui prend une option révolutionnaire ou anarchique et qui ajuste son action en conformité avec ces idéologies se condamne à être minoritaire et à voir son action réduite à une large dose d'inefficacité tant au plan des relations du travail qu'au plan politique.

\section{LE PUBLIC ET LA POLITISATION DES RELATIONS DU TRAVAIL}

Je crois que dans l'ensemble notre population qui est plutôt de tendance assez conservatrice accepte les changements qui se sont effectués dans notre économie avec l'importance grandissante de l'État et la politisation naturelle des relations du travail. Elle accepte aussi le syndicalisme comme agent de négociation collective et instrument de transformation sociale.

Elle trouve normal que les groupements professionnels mènent une action politique auprès de ses membres comme auprès des gouvernements et de l'opinion publique.

Il m'apparaît encore que notre population et même les travailleurs dans leur ensemble sont très réticents vis-à-vis de la politisation des relations du travail lorsque celle-ci est le résultat de l'action systématique de qui que ce soit. Comme la politisation provoquée par les syndicats est plus spectaculaire et atteint plus directement le public dans ses habitudes de penser et surtout dans la satisfaction de ses besoins comme consommateur de biens et de services, il y a danger qu'il se tourne contre les syndicats et exige de l'État un contrôle plus grand de l'action syndicale, les empêchant ainsi de poursuivre leurs objectifs professionnels et même d'être un ferment indispensable dans la société.

\section{POLITIQUES DE MAIN-D'CEUVRE : ÉVALUATION DE L’EXPÉRIENCE QUÉBÉCOISE}

Introduction (JEAN-PAul Deschênes). Les politiques de main-d'oeuvre et le développement socio-économique (PIERre HARvey). Les programmes de maind'œuvre et leur évolution (ÉMILIEN LANDRY et LouIs LEMIEUX). La convention collective et les politiques de main-d'œuvre (Yves DubÉ et JEAN-PAuL DESCHÊNES). Les politiques de main-d'œuvre et la formation professionnelle des adultes (Pierre Paquet). Le maintien du revenu (Gilles Beausoleil). L'évaluation des programmes de main-d'œuvre : résultats et pertinence. La coordination des politqiues de main-d'œuvre (Claude Mérineau).

1 volume, 188 pages - Prix : $\$ 5.50$

LES PRESSES DE L'UNIVERSITÉ LAVAL

G1K 7R4

Québec, P.Q.

Canada 